\title{
The Impact of Housing Features Relative Location on a Turbocharger Compressor Flow
}

\author{
Sidharath Sharma, Martyn L. Jupp, and Simon M. Barrans \\ Turbocharger Research Institute, University of Huddersfield, Huddersfield, United Kingdom \\ Email: \{sidharath.sharma, m.l.jupp, s.m.barrans\}@ @ud.ac.uk \\ Ambrose K. Nickson \\ BorgWarner Turbo Systems, Bradford, United Kingdom \\ Email: knickson@borgwarner.com
}

\begin{abstract}
This work presents an investigation on a flow phenomenon marked by in-plane velocity non-uniformity associated with a ported shroud turbocharger compressor observed upstream of the compressor inlet at lower operating speeds. The effect of structural struts in the ported shroud (PS) cavity and the location of the volute tongue on velocity non-uniformity is studied in this paper by numerically modelling the complete compressor stage using a (Un)steady Reynolds Averaged Navier-Stokes (RANS \& URANS) approach. The results show that the amplitude of in-plane velocity non-uniformity is reduced by removing the struts from the PS cavity. Furthermore, the change in location of the volute tongue is shown to either substantially diminish or enhance the amplitude of velocity nonuniformity based on the relative position of the volute tongue and the struts. The study concludes that the velocity non-uniformity is dependent on the coupled effect of volute tongue and the strut position in the PS cavity..
\end{abstract}

Index Terms-Ported Shroud, Compressor Flow, CFD, RANS, URANS

\section{INTRODUCTION}

Turbocharging of an IC engine increases the specific power output and therefore is heavily used to achieve engine down-sizing and down-speeding. A smaller engine size leads to lower frictional and mechanical losses, improving emissions and thermodynamic efficiency.

The downsized engine is expected to yield a uniform torque curve including high torque at low engine speeds for a good driving experience, specifically in the city driving conditions. This necessitates a wide, stable flow operating region (SFR) for the turbocharger compressor including in the low mass flow region. The phenomenon of surge limits the minimum mass flow, beyond which the compressor cannot be operated safely. A delay in the onset of surge and an enhancement in the stable flow region of compressor operation is achieved either by using active flow control devices or passive flow control devices. Passive flow control works on the principle of recirculating the low momentum fluid to the compressor inlet. Compact, reliable and simple design [1] makes

Manuscript received March 14, 2017; revised April 20, 2017. passive devices the preferred option for automotive applications.

Fisher [1] experimentally investigated a selfrecirculation casing treatment and concluded that the pressure gradient drives the flow out of or into the impeller passages via the ported shroud cavity for near surge and near choke conditions respectively. The results showed increased map width of the compressor at the expense of efficiency. Hunziker et al. [2] investigated the various configurations of a bleed slot using numerical and experimental methods. The optimal design achieved enhanced stable flow region of the compressor without heavily penalising the efficiency.

Semlitsch et al. [3, 4] numerically investigated the ported shroud compressor by employing a scale resolving LES turbulence model to understand the compressor flow field, specifically near surge operation. The results indicated a reduction in the flow disturbances in the blade passage as compared to a non-ported compressor operating near surge, along with an efficiency penalty at off-design conditions due to losses inside the cavity [5] and mixing losses.

The current work investigates the in-plane velocity non-uniformity flow phenomenon associated with the ported shroud design at lower operating speeds by employing a steady RANS and URANS approach. The flow structures at a plane upstream of the PS outlet are investigated for multiple operating speed lines along with the corresponding effect of structural struts and volute tongue location. The impact of the various locations of volute tongue on the velocity non-uniformity is explored, and optimum location of volute tongue relative to struts is quantified.

\section{NUMERICAL MODELLING APPRAOCH}

\section{A. Methodology}

The approach employed in this work is to numerically solve the (un)steady Navier-Stokes equations for each control volume of the computational domain in their conservation form using a commercial CFD coupled solver ANSYS CFX [6]. The full compressor stage is modelled as the flow distortion caused by the volute 
tongue [7] would not be captured using a single blade passage model.

The steady-state RANS approach that is based on the Reynolds averaging of the Navier-Stokes equations along with unsteady RANS is used to directly compute the mean flow solution. The Reynolds stress tensor introduced by the Reynolds averaging formulation is modelled using a two-equation SST closure model. The SST formulation is widely used in turbomachinery CFD problems due to its ability to accurately represent the boundary layer flow and therefore, yields an accurate solution of detaching and swirling flows [8]. The turbulence is assumed to be isotropic with the curvature correction. The working fluid in the compressor is assumed to be a perfect gas with the ideal gas law modelling the fluid behaviour and Sutherland's law approximating the dynamic viscosity. The dependence of specific heat on temperature is modelled by using a temperature polynomial.

The impeller motion is modelled using the Multiple Reference Frame (MRF) approach by employing a frozen rotor interface [6] in the steady-state work as it preserves the circumferential non-uniformity introduced by volute tongue. The use of a single impeller position can be justified as the position of the impeller relative to the volute tongue does not have appreciable impact on compressor performance predictions [9]. Rigid Body Motion (RBM), also known as sliding mesh is used for the URANS model in which the mesh actually rotates every time-step at the transient rotor-stator interface. A steady boundary condition, as a combination of the total pressure at the inlet and mass flow rate at the outlet, is prescribed in this work to model the entire operating range of the compressor including design, choke and near surge conditions. The walls are modelled as smooth with adiabatic and no-slip boundary conditions.

A steady RANS approach has been widely used in literature to model the compressor operation for both design and off-design operating conditions. The recent studies include the works of Yang et al. [10] and Christou [11] presenting a reasonable correlation with experimental data at design as well as near surge conditions. The work of Sivagnanasundaram et al. [12-14] used steady RANS to analyse the PS flow features for the compressor operating near surge. Although the RANS approach is expected to yield poor predictions for the near surge condition owing to the comparable order of the mean and turbulent time scales [15], but in the current work, the operation of compressor at the design condition is of primary interest. Therefore, the use of steady RANS as the primary computational approach is well justified for this work. Furthermore, URANS approach is used for one operating point to validate the flow features predicted by the steady model.

\section{B. Geometry and the Computational Model}

A commercially-available turbocharger compressor geometry with a ported shroud (PS) feature as shown in Fig. 1 is used in this investigation. The PS cavity is structurally supported by three struts in a symmetric arrangement. The impeller backside cavity (IBC) has been modelled in this investigation. The specification of the compressor is provided in Table I.

The computational domain is spatially discretized by an unstructured polyhedral grid that is generated from the tetrahedral cells by the vertex-centered numerical approach in ANSYS CFX [6]. A polyhedral mesh is less diffusive and offers significant accuracy gains compared to an equivalent size unstructured grid of other cell types (i.e. tetrahedral) [16]. Furthermore, a polyhedral grid is expected to offer higher accuracy over equivalent hexahedral grid in the cases where secondary flows are relevant [17] and therefore, both design and off-design operating conditions can be accurately modelled using a polyhedral grid. The time integration of temporal form is performed using an implicit second order scheme for the URANS model in which the time advances with each iteration. The time step for advancement is chosen in such a way that the impeller mesh turns by $4^{\circ}$ per time step at a specific operating speed. Five inner coefficient loops are used for each iteration to achieve the convergence of residuals up to four orders $\left(10^{-4}\right)$.

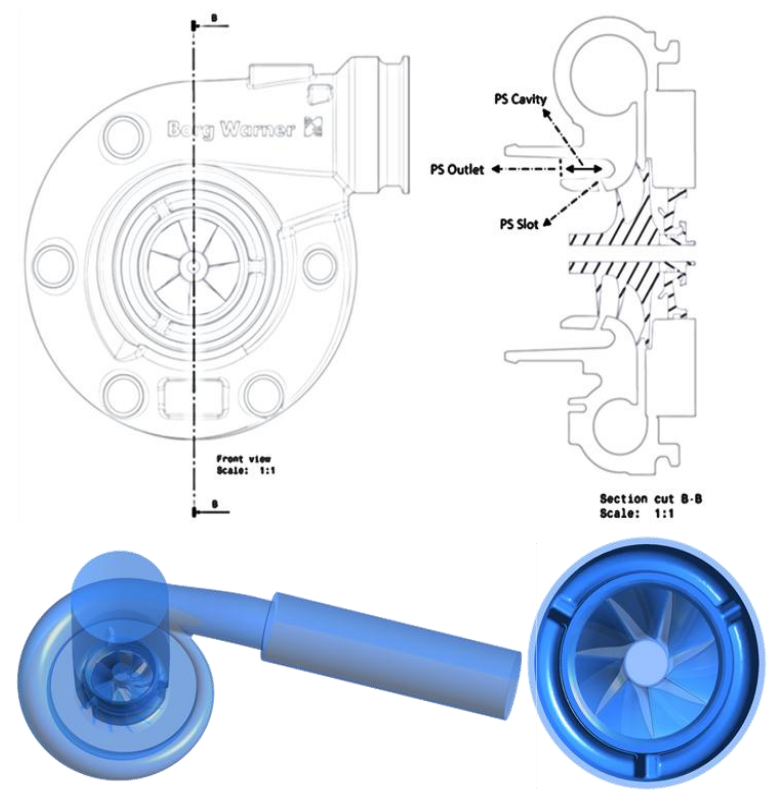

Figure 1. Compressor geometry schematics showing the various features of the PS

The inlet and outlet sections of the compressor are extended by 2.5 exducer diameter $\left(2.5 D_{e}\right)$ upstream and downstream for the RANS model and $6 D_{e}$ downstream for the URANS model to decrease the impact of the boundary conditions on the mean flow as well as to capture the flow instability that would be propagated upstream of impeller inlet in off-design conditions. The numerical set-up is not the perfect replication of the experimental set-up but is designed for computational efficiency.

The computational domain is assessed to yield the flow solution independent of grid density. The near-wall grids are investigated for their ability to resolve boundary layer flow by achieving a value of $y^{+}$closer to unity. Fig. 2 
shows the global contours of $y^{+}$values along with the $y^{+}$ distribution at the impeller surface. The chosen grid of steady state model consists of approximately 15 million cells and the boundary layer region is modelled using 12 prism layers growing exponentially with a growth ratio of 1.3. The turbulence numerics are modeled using a high resolution scheme [6] which uses a second order advection scheme and the combination of a second order and a first order backward Euler scheme to maintain a bounded solution. The numerical model uses $1 \%$ turbulent intensity and a turbulent viscosity ratio of 10 . The operation of the compressor at various speeds shown in Table II is simulated across the mass flow range from surge at one end to choke at another.

TABLE I. COMPRESSOR SPECIFICATIONS

\begin{tabular}{ll}
\hline TC design intent & Commercial vehicles \\
\hline Power & $400-600 \mathrm{~kW}$ \\
Number of blades & $7+7$ (main and splitter) \\
Inducer to exducer ratio & 0.67 \\
Diffuser area ratio & 0.62 \\
\hline
\end{tabular}

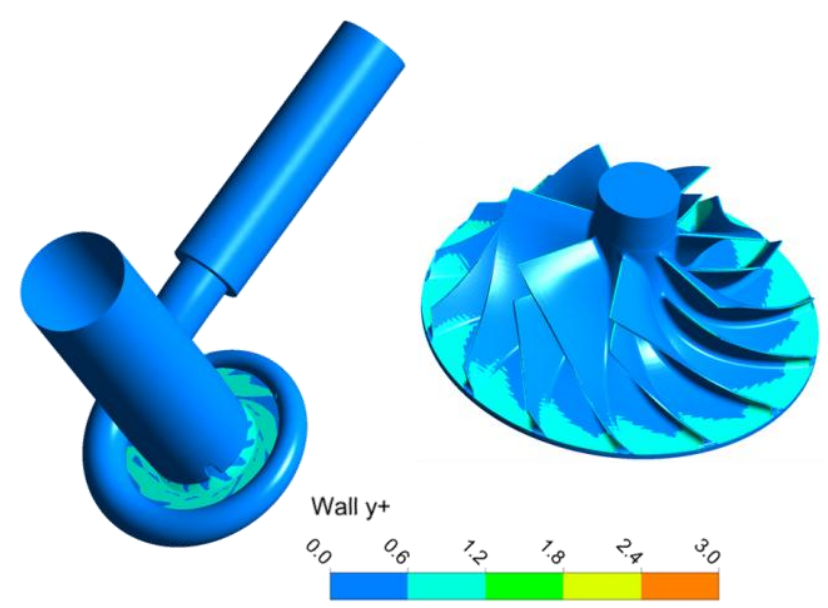

Figure 2. Global and impeller $y+$ contours of the numerical grid

\section{Validation of the Numerical Model}

The computational model is validated by comparing the numerical results with gas stand measurement data. The total-to-total pressure ratio $\Pi_{t, t}$ and isentropic efficiency $\eta_{s}$ are plotted against air mass flow on a compressor performance map shown in Fig. 3. The fluctuations of the compressor flow solution, as predicted by steady RANS approach at various operating points, is displayed in the form of error bars in Fig. 3. The vertical error bar represents the fluctuation of the ordinate i.e. pressure ratio/isentropic efficiency and the horizontal bar indicates the variation in abscissa i.e. mass flow rate. The error bars are small (barely visible) at the design condition implying flow is approximately steady as opposed to the increased error bar sizes indicating unsteady nature of the flow near surge. The numerical predictions and the experimental measurements show good agreement at low speeds although the deviation in results increases at higher speeds as seen by Zheng [9].

TABLE II. OPERATING LiNES AND POINTS DETAILS

\begin{tabular}{ccc}
\hline \multicolumn{1}{c}{ Iso-speed line } & RPM & Design mass flow \\
\hline Reference speedline (RSL) & $N_{\text {ref }}$ & $\dot{m}_{\text {ref }}$ \\
Speedline 1 (SL1) & $0.8 N_{\text {ref }}$ & $0.8 \dot{m}_{\text {ref }}$ \\
Speedline 2 (SL2) & $1.2 N_{\text {ref }}$ & $1.2 \dot{m}_{\text {ref }}$ \\
\hline
\end{tabular}

The numerical performance parameters under-predict which seems counter intuitive as the walls are modelled as smooth and adiabatic. The work of Zhigang et al. [18] shows that modeling the IBC results in the lower values of the performance variables computed in the numerical model. Isentropic efficiency predictions show a higher deviation from the measured data specifically at the choke condition compared to the pressure ratio predictions.

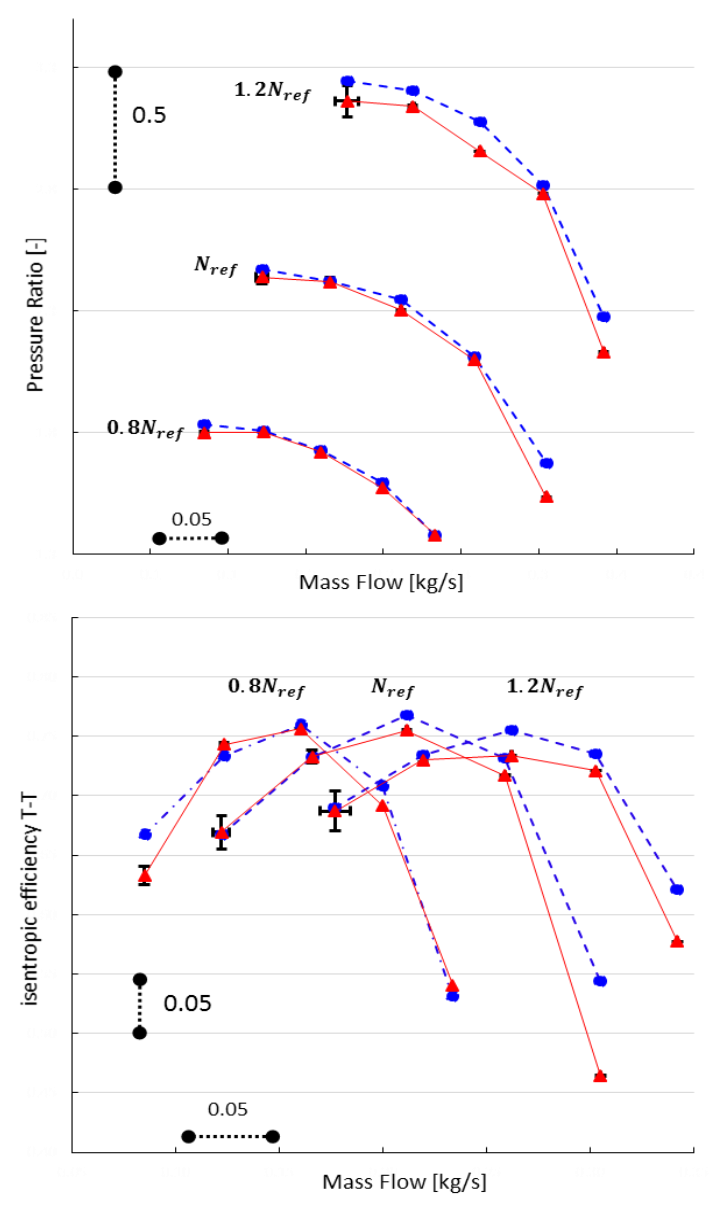

Figure 3. Pressure T-T and isentropic efficiency map: numerical results (red solid line) are compared to gas stand measurements (blue dashed line)

Efficiency values are calculated using two numerically computed variables $p_{o u t, 0}$ and $T_{o u t, 0}$ while pressure ratio 
is dependent only on the $p_{\text {out }, 0}$ and therefore, the efficiency values include a compounded error compared to pressure ratio. Nevertheless, the computed results are considered acceptably close to the gas stand measurements and consistently capture the pressure ratio and isentropic efficiency trends.

\section{ANALYSIS OF PORTED SHROUD OPERATION}

The increase in the stable flow region achieved using ported shroud design comes with a penalty in efficiency along with deteriorated acoustics performance [19]. The interaction of the flow exiting the PS outlet with the incoming flow leads to turbulent mixing zones [4]. The frictional losses in the PS cavity and the mixing losses are hypothesised to have a significant effect on the performance of the PS compressor [4, 5, 11]. Furthermore, the turbulent mixing can be a possible contributor of broadband noise increase that is usually seen with the use of PS design [19].

\section{A. Flow in the PS Cavity}

The fraction of compressor inlet flow that is recirculating through the PS cavity $\dot{m}_{p_{s}} / \dot{m}$ is investigated at multiple operating conditions for three different isospeed lines $\left(0.8 N_{\text {ref }}, N_{\text {ref }}\right.$ and $\left.1.2 N_{\text {ref }}\right)$ that are plotted in Figure 4 to understand the initiation of mixing process. The data shows a significant fraction of the compressor inlet mass flow is recirculated through the PS cavity for near surge operating conditions. The primary aim of the current work is to understand the impact of the ported shroud feature at the design operating condition. Although the recirculated flow through the PS cavity at the design operating condition is relatively small compared with near surge conditions, it is interesting to note that the flow is still recirculated from the blade passages to the impeller inlet, at the design condition when the compressor is operating at lower speed $\left(0.8 N_{\text {ref }}\right)$ and the flow is added into the blade passages via the PS slot for higher operating speed $\left(1.2 N_{\text {ref }}\right)$. Therefore mixing losses at the design conditions are expected at lower operating speeds while frictional losses can be expected at both lower and higher operating speeds.

\section{B. Velocity Non-uniformity Upstream of PS Outlet}

To further investigate the characteristics of flow exiting PS cavity, in-plane velocities on a plane upstream of PS outlet is studied for the three iso-speed lines using a steady state approach. Figure 5 shows a non-uniformity in the in-plane velocity contours for the lower operating speeds i.e. $0.8 N_{\text {ref }}$ and $N_{\text {ref }}$ while the velocity at the higher speed $\left(1.2 N_{\text {ref }}\right)$ is fairly symmetric. The velocity non-uniformity is located in angular proximity of the volute tongue between supporting strut 1 and strut 2 . The in-plane velocity contours predicted by the steady approach are further validated using a transient (URANS) sliding mesh approach for the design condition at $N_{\text {ref }}$ speed. The in-plane velocity contours for every $24^{\circ}$ of rotation of the impeller (approximately half blade passing) for four successive periods, are shown in Fig. 6. The velocity non-uniformity predictions computed by the unsteady transient approach further reinforces the conclusion that it is an aerodynamic phenomenon rather than a spurious result due to the fixed position frozen rotor approach. The steady approach is therefore used thereafter in this work.

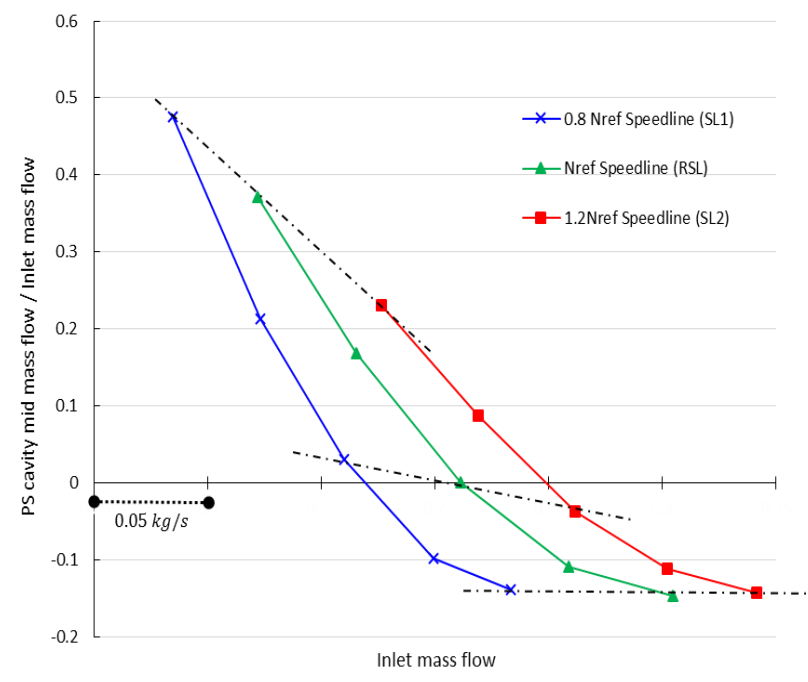

Figure 4. The fraction of inlet flow recirculating through the PS cavity for various operating points

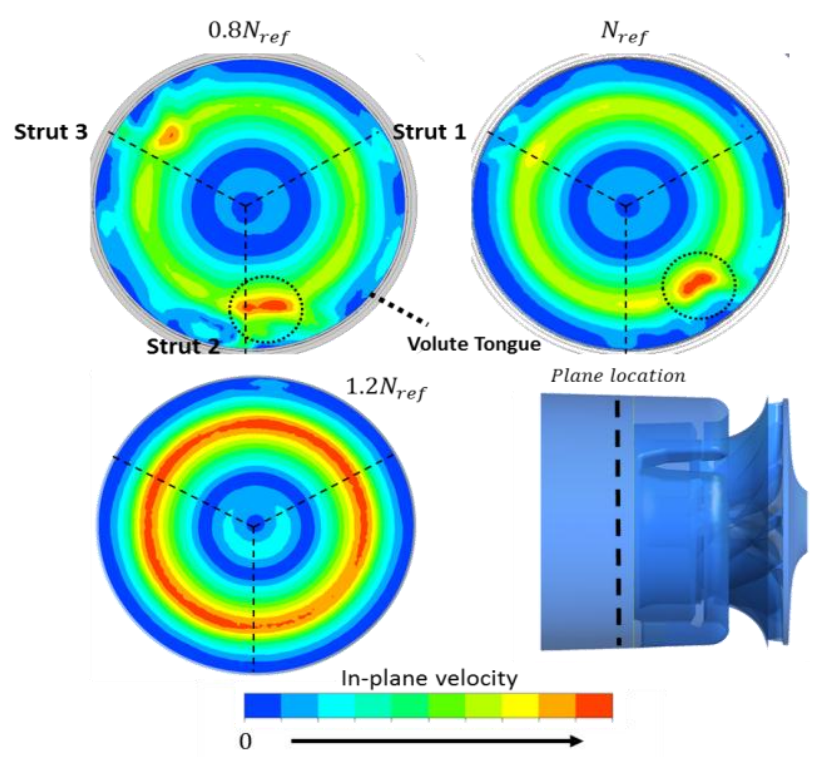

Figure 5. In-plane velocity contours a plane $3 \mathrm{~mm}$ upstream of PS outlet for design operating condition

\section{1) Effect of supporting struts}

The flow leaves the PS cavity at PS outlet in the form of jets [11] corresponding to the struts. Therefore the impact of supporting struts is further investigated. The structural supporting struts are removed from the numerical model, and the flow solution is computed using the steady approach for the design condition at $N_{\text {ref }}$ speed. The in-plane velocity contours for the model with 
and without supporting struts are compared in Fig. 7. The results show that the amplitude of velocity nonuniformity is slightly reduced by removing the struts from the PS cavity. This suggests the role of other asymmetries in the model i.e. the volute tongue on velocity non-uniformity.

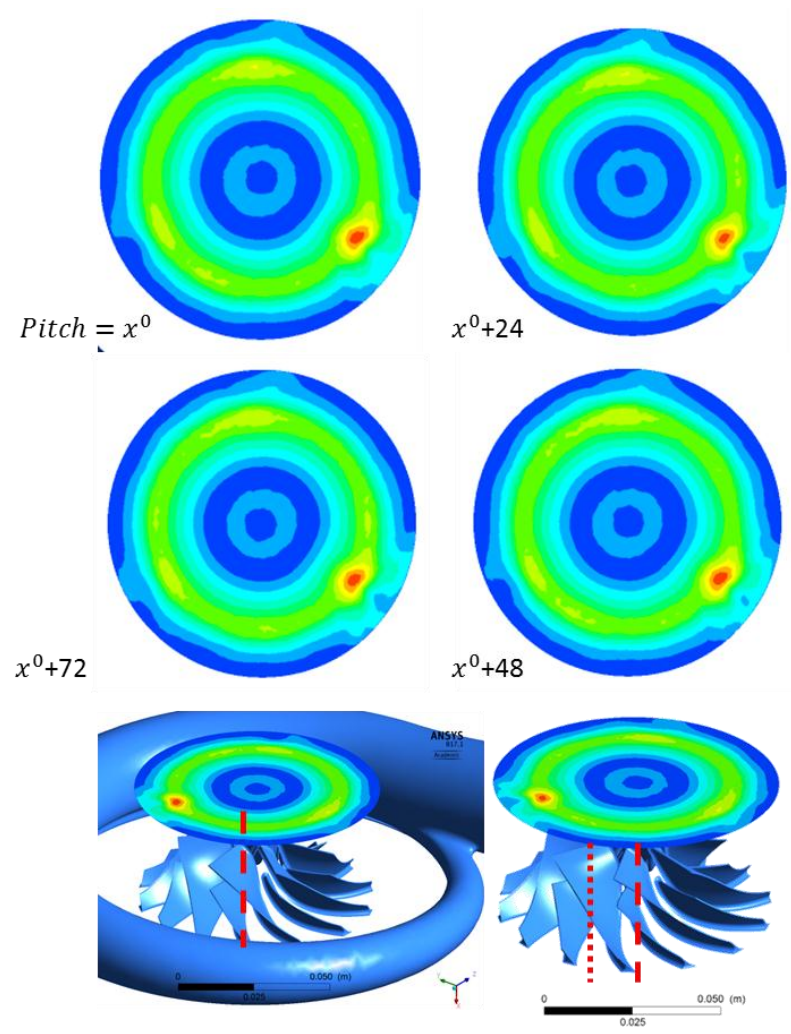

Figure 6. In-plane velocity contours at design condition of $\mathrm{N}_{\text {ref }}$ for various position of impeller

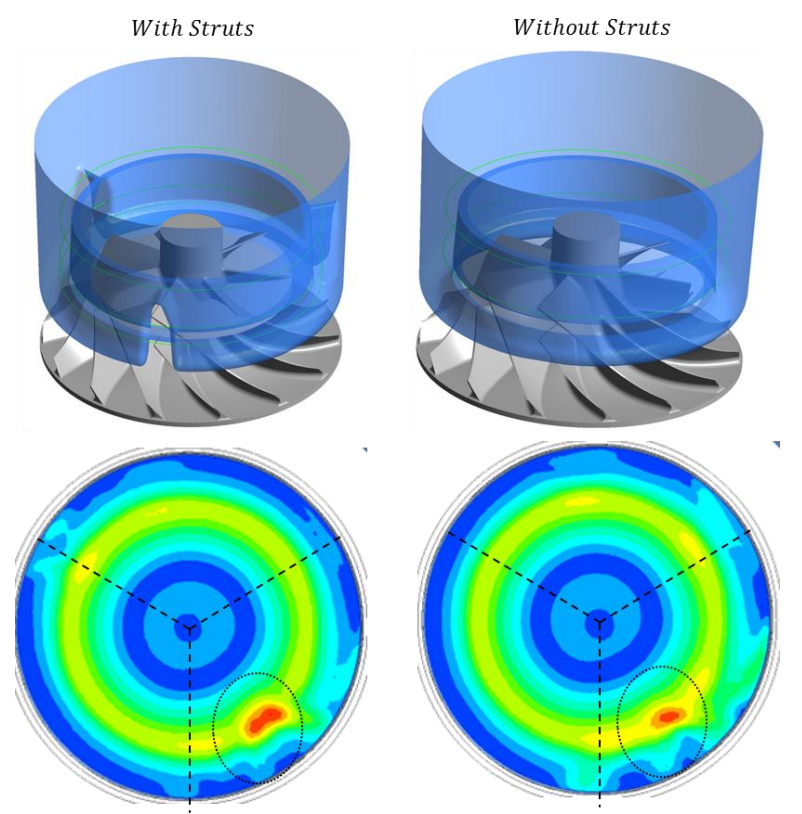

Figure 7. Comparison of in-plane velocity contours of the model with and without struts for the Nref design point

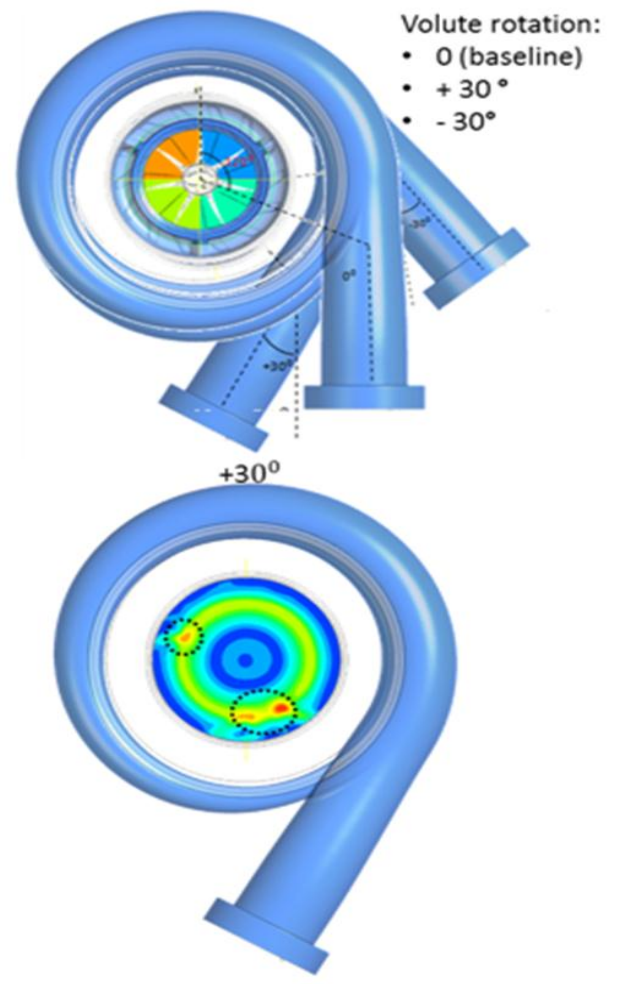

$\mathbf{0}^{\circ}$

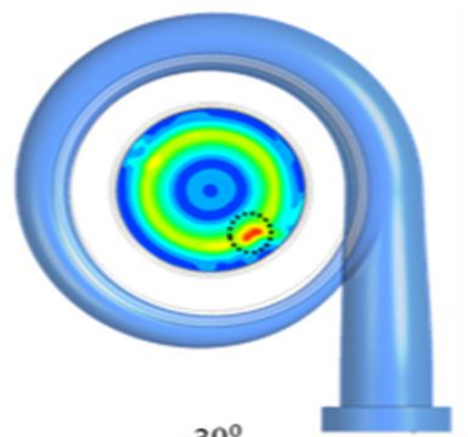

$-30^{\circ}$

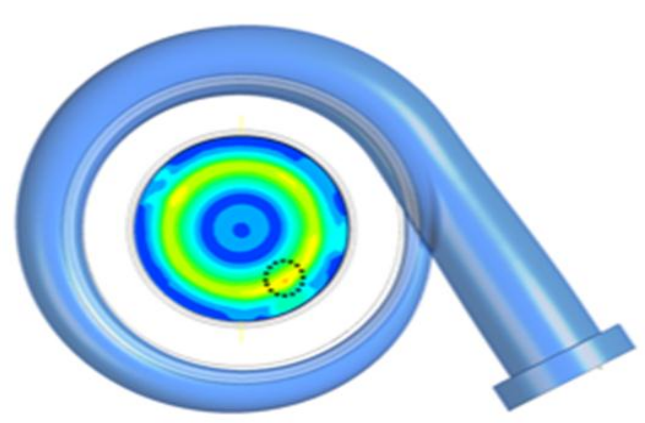

Figure 8. Volute tongue positions and computed in-plane velocity contours at the plane $3 \mathrm{~mm}$ upstream of PS outlet

2) Impact of volute tongue location

The impact of the volute tongue position on the velocity non-uniformity is investigated by changing the position of the volute tongue by $30^{\circ}$ in clockwise $\left(+30^{\circ}\right)$ and counter clockwise $\left(-30^{\circ}\right)$ direction in the baseline model (with supporting struts). Fig. 8 shows the various tongue positions and their computed in-plane velocity contours. The results clearly show that the clockwise 
rotation $\left(+30^{\circ}\right)$ of the volute increases the amplitude of the velocity non-uniformity as well as introduces an additional non-uniformity near strut 3 .

The in-plane velocity non-uniformity is almost completely eliminated with the counter clockwise $\left(-30^{\circ}\right)$ rotation of the volute. The sole effect of the volute tongue on velocity non-uniformity at the compressor inlet is further investigated by removing the structural struts from the numerical model showing the least (suppressed) velocity non-uniformity i.e. with volute rotated counter clockwise by $-30^{\circ}$. Fig. 9 displays the comparison of the volute counter clockwise rotated model with and without struts. The velocity non-uniformity reappears when the struts are removed, directing that the suppression of velocity non-uniformity is a coupled effect of structural struts and volute tongue. Therefore the location of volute tongue relative to PS struts is important to suppress the non-uniformity at the compressor inlet leading to reduced mixing losses.
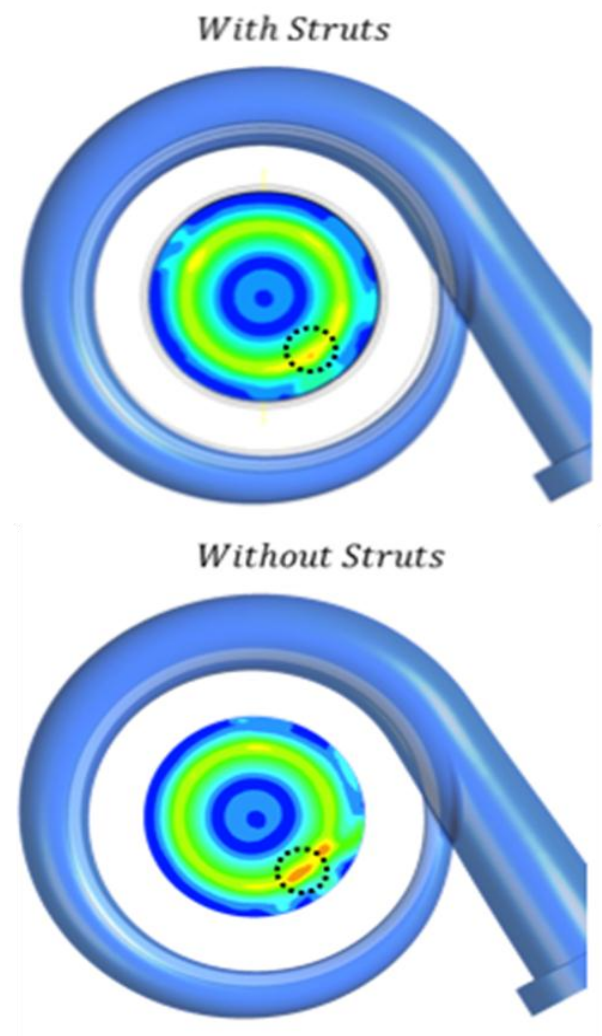

Figure 9. In-plane velocity contours of the modified volute tongue model with and without struts

\section{CONCLUSIONS}

The velocity non-uniformity associated with the ported shroud turbocharger compressor operating at the design conditions of lower speeds is investigated in this study using the steady RANS and URANS approach. The performance parameters i.e. isentropic efficiency and pressure ratio predicted by the numerical model are in good agreement with the experimental values measured on a gas stand test rig. The computed results consistently capture the pressure ratio and isentropic efficiency trends.
The investigation of the in-plane velocity field upstream of PS outlet shows velocity non-uniformity between struts 1 and 2 at lower operating speeds. The transient URANS prediction further reinforces the existence of velocity non-uniformity.

Analysis of the flow after removing the structural struts shows slightly reduced velocity non-uniformity, and counter-clockwise rotation of the volute tongue substantially eliminates it. Velocity non-uniformity resurfaces when the struts are removed from the rotated volute model confirming the location of the volute tongue relative to struts as the critical condition for the suppression of velocity non-uniformity.

\section{FUTURE WORK}

Investigation on the mechanism responsible for the non-uniformity is being explored. The impact of volute tongue relative location on the compressor flow field at the near surge operating conditions is planned to be investigated.

The effect of the velocity non-uniformity on the compressor inlet noise characteristics is planned to be investigated experimentally.

\section{ACKNOWLEDGMENT}

The project is supported the Regional Growth Fund (RGF Grant Award 01.09.07.01/1789C). The authors would like to thank BorgWarner Turbo Systems for permission to publish the results presented in this paper. The support of the HPC group at the University of Huddersfield is gratefully acknowledged.

$\begin{array}{cl} & \text { NOMENCLATURE } \\ \text { PS } & \text { Ported shroud } \\ \text { IC } & \text { Internal combustion } \\ N_{r e f} & \text { Reference speedline } \\ \dot{m}_{p s} & \text { Mass flow rate at the specified } \\ D_{e} & \text { location i.e. PS } \\ \Pi_{t-t} & \text { Exducer diameter, } \mathrm{m} \\ \eta_{s} & \text { Total to total pressure ratio } \\ y^{+} & \text {Isentropic efficiency } \\ T_{\text {out }, 0} & \text { Dimensionless wall distance } \\ p_{\text {out }, 0} & \text { Total temperature at the outlet }\end{array}$

\section{REFERENCES}

[1] F. B. Fisher, "Application of Map Width Enhancement Devices to Turbocharger Compressor Stages," 1988.

[2] R. Hunziker, H. P. Dickmann, and R. Emmrich, "Numerical and experimental investigation of a centrifugal compressor with an inducer casing bleed system," in Proc. the Institution of Mechanical Engineers, Part A: Journal of Power and Energy, vol. 215, pp. 783-791, 2001.

[3] B. Semlitsch and M. Mihăescu, "Flow phenomena leading to surge in a centrifugal compressor," Energy, vol. 103, pp. 572-587, 5/15/ 2016. 
[4] B. Semlitsch, V. Jyothishkumar, M. Mihaescu, L. Fuchs, E. Gutmark, and M. Gancedo, "Numerical flow analysis of a centrifugal compressor with ported and without ported shroud," SAE Technical Papers, vol. 1, 2014.

[5] H. Chen and V. M. Lei, "Casing treatment and inlet swirl of centrifugal compressors," Journal of Turbomachinery, vol. 135, p. 041010, 2013.

[6] A. C. A. Research, ANSYS CFX-Solver Theory Guide. USA: ANSYS, Inc., 2013.

[7] H. Chen, S. Guo, X. C. Zhu, Z. H. Du, and S. Zhao, "Numerical simulations of onset of volute stall inside a centrifugal compressor," in Proc. the ASME Turbo Expo, 2008.

[8] F. R. Menter, "Two-equation eddy-viscosity turbulence models for engineering applications," AIAA Journal, vol. 32, pp. 1598-1605, 1994/08/01 1994.

[9] X. Q. Zheng, J. Huenteler, M. Y. Yang, Y. J. Zhang, and T. Bamba, "Influence of the volute on the flow in a centrifugal compressor of a high-pressure ratio turbocharger," in Proc. the Institution of Mechanical Engineers, Part A: Journal of Power and Energy, vol. 224, pp. 1157-1169, December 1, 2010.

[10] M. Yang, R. Martinez-Botas, Y. Zhang, and X. Zheng, "Effect of self-recirculation-casing treatment on high pressure ratio centrifugal compressor," Journal of Propulsion and Power, pp. 1-9, 2016.

[11] G. A. Christou, "Fluid mechanics of ported shroud centrifugal compressor for vehicular turbocharger applications," Ph. D., Massachusetts Institute of Technology. Department of Aeronautics and Astronautics., Massachusetts Institute of Technology, Massachusetts, 2015.

[12]S. Sivagnanasundaram, S. Spence, J. Early, and B. Nikpour, "Experimental and numerical analysis of a classical bleed slot system for a turbocharger compressor," in Proc. IMechE 10th International Conference on Turbochargers and Turbocharging, ed, 2012, pp. 325-341.

[13] S. Sivagnanasundaram, S. Spence, J. Early, and B. Nikpour, "An impact of various shroud bleed slot configurations and cavity vanes on compressor map width and the inducer flow field," vol. 7, ed, 2011, pp. 2009-2021.

[14] S. Sivagnanasundaram, S. Spence, and J. Early, "Map width enhancement technique for a turbocharger compressor," Journal of Turbomachinery, vol. 136, pp. 061002-061002, 2013.

[15] D. C. Wilcox, Turbulence Modeling for CFD vol. 2: DCW industries La Canada, CA, 1998.
[16] P. Chow, M. Cross, and K. Pericleous, "A natural extension of the conventional finite volume method into polygonal unstructured meshes for CFD application," Applied Mathematical Modelling, vol. 20, pp. 170-183, 1996/02/01 1996.

[17] M. Tritthart and D. Gutknecht, "Three-dimensional simulation of free-surface flows using polyhedral finite volumes," Engineering Applications of Computational Fluid Mechanics, vol. 1, pp. 1-14, 2007/01/01 2007.

[18] Z. Sun, C. Tan, and D. Zhang, "Flow field structures of the impeller backside cavity and its influences on the centrifugal compressor." vol. 7, ed, 2009, pp. 1349-1360.

[19] H. Chen and J. Yin, "Turbocharger compressor development for diesel passenger car applications A2 Group, Institution of Mechanical Engineers Combustion Engines \& Fuels," in Proc. 8th International Conference on Turbochargers and Turbocharging, ed: Woodhead Publishing, 2006, pp. 15-27.

Sidharath Sharma is a Ph.D. researcher with Turbocharger Research Institute. He received B.S. in Mechanical Engineering from National Institute of Technology, Srinagar, India. Prior to enrolling at TRI, he worked in Powertrain NVH and acoustics. Sidharath's research interests include computational aeroacoustics and turbulence modelling.

M. L. Jupp was born in the United Kingdom. He received his BEng in mechanical engineering from De Montfort University $\mathrm{UK}$ and $\mathrm{PhD}$ in aerodynamics from Glasgow University, UK. $\mathrm{He}$ is currently a Principle Enterprise Fellow in the Turbocharger Research Institute at the University of Huddersfield.

Simon M. Barrans was born in the United Kingdom. He received his first degree in Nuclear Engineering from Manchester University UK and $\mathrm{PhD}$ in finite element analysis of boundary stress predictions from the University of Huddersfield, UK. He is currently working as Reader within the Turbocharger Research Institute at the University of Huddersfield.

A. K. Nickson was born in the United Kingdom. He received his BEng in mechanical engineering and $\mathrm{PhD}$ in non-Newtonian fluid mechanics from Liverpool University UK. He is currently a Staff Performance Engineer at BorgWarner Turbo Systems. 